Jpn. J. Med. Sci. Biol., 48, 163 - 175, 1995.

\title{
NON-RADIOACTIVE MISMATCH ANALYSIS TO DETECT SMALL MUTATIONS IN HUMAN HYPOXANTHINE-GUANINE PHOSPHO- RIBOSYL TRANSFERASE CDNA
}

\author{
Koji TSUBOI, Tadao NOSE, Richard T. OKINAKA1 and David J. CHEN1
}

Institute of Clinical Medicine, University of Tsukuba, Tsukuba Science City, Ibaraki 305 and 1 Life Sciences Division, Los Alamos National Laboratory, Los Alamos, NM 87545, USA

(Received December 16, 1994. Accepted May 31, 1995)

SUMMARY: We have combined a cDNA-driven PCR technique and a nonradioactive chemical-cleavage mismatch method, followed by a direct sequencing for detecting small mutations in the human hypoxanthine-guanine phosphoribosyl transferase (HPRT) gene. HPRT cDNA was synthesized by RT-PCR from 1,000 wild-type or HPRT(- ) mutant cells. Wild-type cDNA was hybridized with mutant $\mathrm{cDNA}$ to form heteroduplexes. The resultant mismatched bases were modified and cleaved by base-specific chemicals, followed by analysis by denaturing polyacrylamide gel electrophoresis. Cleaved fragments were detected without using radioactive materials. Finally, direct sequencing of the PCR products was performed with a focus on a small limited region indicated by the mismatch analysis (focused sequencing). In this study, three small mutations in exon-3 of HPRT cDNA were detected and characterized completely with this system. As compared with the radioactive method, this system was shown to be very simple and efficient.

\section{INTRODUCTION}

The X-linked hypoxanthine-guanine phosphoribosyl transferase (HPRT) gene has been utilized extensively as a marker for the analysis of mutations induced by physical and chemical carcinogens in both human and rodent cells. One

坪井康次·能勢忠男(筑波大学臨床医学系脳神経外科 つくば市天王台1-1-1) 
concern in the analysis of the HPRT gene is detecting point mutations to identify "hot-spots". Although various direct nucleotide sequencing approaches have been attempted (1-5), it seemed difficult to locate single point mutations within the more than 700 bases that constitute the coding region (cDNA) of the HPRT gene. Under these circumstances, it would be desirable to have a simple prescreening strategy that could detect and locate the position of mutations before direct nucleotide sequencing is attempted. In this report we describe an approach that couples an RT-PCR (reverse transcription - polymerase chain reaction) amplification method to a non-radioactive DNA-DNA mismatch hybridization technique. Final characterization of the detected mutations was performed by direct nucleotide sequencing with a focus on a small limited region indicated by the prior mismatch analysis (focused sequencing).

\section{MATERIALS AND METHODS}

Cell culture: Primary human skin fibroblasts (HSF) obtained from newborn foreskins were prepared as reported (6) and used as wild-type controls. Three HPRT-deficient mutants isolated in our institute from X-ray irradiated populations of the human B-lymphoblastoid cell line, TK6, were used to validate our present technical approach. These mutants were known to have noncharacterized small mutations in exon-3 of the HPRT gene. Both the mutant and HSF cells were cultured in Dulbecco's modified Eagle's medium (DMEM) supplemented with $100 \mu \mathrm{g} / \mathrm{ml}$ streptomycin, $100 \mathrm{units} / \mathrm{ml}$ penicillin and $10 \%$ fetal calf serum (FCS). The cultures were maintained at $37 \mathrm{C}$ in a humidified incubator with $5 \% \mathrm{CO}_{2}$.

RT-PCR: First strand cDNA synthesis and subsequent PCR amplification were performed by the method of Yang et al (4). Briefly, 1,000 HSF or mutant lymphoblast cells were microfuged at $15,000 \mathrm{rpm}$ for $1 \mathrm{~min}$. These cell-pellets were directly incubated at $37 \mathrm{C}$ for $1 \mathrm{hr}$ in $5 \mu \mathrm{l}$ of the first strand cDNA synthesis cocktail to be used as cDNA templates in the subsequent PCR reaction. The first PCR was performed in 50- $\mu$ l volume with a thermal cycler (Perkin-Elmer Cetus, Norwalk, CT) under the conditions of Yang et al. (4) using an Ampli-Taq (PerkinElmer Cetus). After the first pair of primers (primers 1 and 2) were removed on Sepharose columns (CL-6B, Pharmacia, Uppsala, Sweden), $1 \mu$ l of the product was used as a template for the second series of PCR. The conditions were identical to those of the initial PCR reaction except for the use of a nested pair of primers (see primers 3 and 4 below). The sequences of the primers were as follows: [the num- 
bering system is as described by Jolly et al. (7) for HPRT cDNA with the AUG start codon numbered at bp 86].

\section{Primer 1: (38) 5' CTCTGCTCCGCCACCGGCTTCCT 3' (60) \\ Primer 2: (975) 5' CCGCCCAAAGGGAACTGATAGTC 3' (953) \\ Primer 3: (64) 5' CCTGAGCAGTCAGCCCGCGC 3' (83) \\ Primer 4: (821) 5' GATAATTTTACTGGCGATGT 3' (801)}

These primers were synthesized based on the information provided by Yang et al. (4) and Jolly et al (7). The final products were fractionated by $0.8 \%$ agarose gel electrophoresis and visualized by ethidium bromide staining. Appropriate fragments were eluted from the gel matrix with Prep-A-Gene (Bio-Rad, Hercules, $\mathrm{CA}$ ) at a final concentration of $50 \mathrm{ng} / \mu \mathrm{l}$.

Non-radioactive chemical mismatch analysis: Wild-type and mutant cDNAs (500 ng each) were mixed in $50 \mu \mathrm{l}$ of the hybridization mix containing $0.3 \mathrm{M} \mathrm{NaCl}$, $3.5 \mathrm{mM} \mathrm{MgCl}_{2}$, and $3 \mathrm{mM}$ Tris-HCl, $\mathrm{pH}$ 7.7. Mixtures in tubes were heated to 100 $\mathrm{C}$ for $5 \mathrm{~min}$ and incubated at $42 \mathrm{C}$ for more than $3 \mathrm{hr}$. Based on the method of Cotton et al. (11), one-half of each heteroduplex solution $(25 \mu \mathrm{l})$ was treated with $20 \mu \mathrm{l}$ of 2.5\% pyridine (Sigma Chemical Co., St. Louis, MO) in water and $5 \mu \mathrm{l}$ of $1 \%$ osmium tetroxide (OT) (Aldrich Chemical Co., Inc., Milwaukee, WI) in water. The final concentration of OT was $0.1 \%$. The mixture was vortexed and incubated at $37 \mathrm{C}$ for $15 \mathrm{~min}$. The remaining heteroduplex solution $(25 \mu \mathrm{l})$ was treated with $100 \mu \mathrm{l}$ of $5 \mathrm{M}$ hydroxylamine hydrochloride (HH) (Sigma Chemical Co.) titrated to pH 6.0 by diethylamine (Sigma Chemical Co.). The mixture was vortexed and incubated at $37 \mathrm{C}$ for $20 \mathrm{~min}$. Then, $150 \mu \mathrm{l}$ of ice-cold water was added to stop the reactions and the resultant chemically modified DNAs were purified with PrepA-Gene (Bio Rad) in a final volume of $20 \mu$ l. Ten microliters of freshly prepared $30 \%$ piperidine (Sigma Chemical Co.) was then added to each tube (final concentration 10\%) and incubated at $90 \mathrm{C}$ for $20 \mathrm{~min}$. After ethanol precipitation in the presence of $0.8 \mathrm{M}$ ammonium acetate and $20 \mu \mathrm{g}$ of mussel glycogen (GIBCO BRL, Gaitherburg, MO), the pellets were rinsed once with $70 \%$ ethanol and dried completely. The samples were analyzed by electrophoresis on a denaturing polyacrylamide gel, and the DNA was visualized by staining with $0.5 \mu \mathrm{g} / \mathrm{ml}$ ethidium bromide.

Radioactive chemical mismatch analysis: The ordinary radioactive technique was used for comparison with our non-radioactive mismatch technique. The preparation of radioactive wild type-probe and the subsequent chemical reactions were identical to the method mentioned by Cotton et al. (8) and Gormpe et al (9). Briefly, the individual nested primers (primer-3 and -4) were end-labeled according to the standard protocols with T4 polynucleotide kinase (21), in order to generate strand specifically radiolabeled wild-type DNA probes. The labeled primers were purified on spin columns (Bio-Gel P-30, Bio Rad) and each "hot" primer was used to drive individual PCR reactions to produce DNA probes labeled in either the "plus" or "minus" strands. Wild-type/mutant heteroduplexes were formed by mixing $10 \mathrm{ng}$ of the radiolabeled probe DNA with $100 \mathrm{ng}$ of target DNA 
in the same reactions mentioned above. Each target DNA was melted and reannealed to both strand-specific probes in separate reactions. The following chemical modification and cleavage of mismatched bases are identical to those described in the non-radioactive method. Ten thousand counts per minute of each final product was fractionated by electrophoresis on sequencing gels followed by detection of specific products by autoradiography.

Direct sequencing: Sequencing was performed by a modified Sanger's dideoxy method using a Bst sequencing kit obtained from Bio Rad. Purified PCR products (0.5-0.7 pmoles) were mixed with 2-5 pmoles of sequencing primer (268ATGGGAGGCCATC-280) and $2 \mu \mathrm{l}$ of buffer supplied by the manufacturer in 15 $\mu \mathrm{l}$ of a reaction volume. The mixtures were heated to $95 \mathrm{C}$ for $5 \mathrm{~min}$ and snapcooled immediately in an ethanol-dry-ice bath for $5 \mathrm{~min}$. One microliter of $[\alpha-$ 32P]-dATP (NEN Dupont, Wilmington, DE) and 1 unit of Bst polymerase (Bio Rad) were added to this annealing mix on ice. The extension-termination reaction was performed according to the manufacturer's standard protocol with their dNTP-ddNTP mix. After the chase reaction, 2 units of terminal deoxynucleotidyl transferase (TdT) (USB, Cleveland, $\mathrm{OH}$ ) in $1 \mu \mathrm{l}$ of a solution containing $0.8 \mu \mathrm{l}$ of $1 \mathrm{mM}$ dNTPs in $1 \times \mathrm{TdT}$ buffer was added to each tube and incubated at $37 \mathrm{C}$ for $30 \mathrm{~min}$ according to the method of Fawcett et al (10). After adding the formamide-loading solution, samples were analyzed by sequencing gel (5\%) electrophoresis followed by autoradiography.

\section{RESULTS}

\section{RT-PCR (Fig. 1)}

Products formed by the initial PCR amplification show faint bands at approximately $920 \mathrm{bp}$ in the wild-type and one of the mutant lines. After a second 30 rounds of PCR amplification with "nested" primers, a large amount of the 740 bp product was observed in all of the samples. The amount of the $740 \mathrm{bp}$ fragment in mutant-3, however, was substantially reduced and a $<600$ bp major product appeared. The $740 \mathrm{bp}$ fragments of all samples, including mutant-3, were purified on preparative gels for further analysis. In addition, the smaller fragment ( $<600 \mathrm{bp}$ ) of mutant-3 was also purified for characterization.

\section{Non-radioactive Chemical Mismatch Analysis (Fig. 2)}

Cleavage products were detected by ethidium bromide staining when the initial amount of DNA for heteroduplex formation was greater than $500 \mathrm{ng}$. No signal was detected when the initial amount of DNA was under $300 \mathrm{ng}$ (data not 


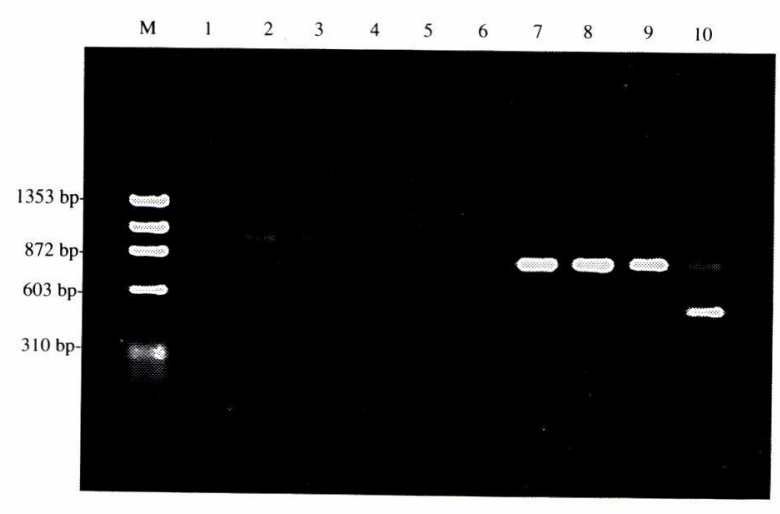

Fig. 1. RT-PCR of the HPRT gene. M: $\Phi$ X-Hae III digest. Lanes 1-5: products after initial 30 cycles of PCR. 1: control (no cell), 2: wild-type, 3: mutant-1, 4: mutant-2, 5: mutant-3. Lanes 6-10: products after second run of PCR. Materials in lanes 6-10 correspond to lanes 1-5.

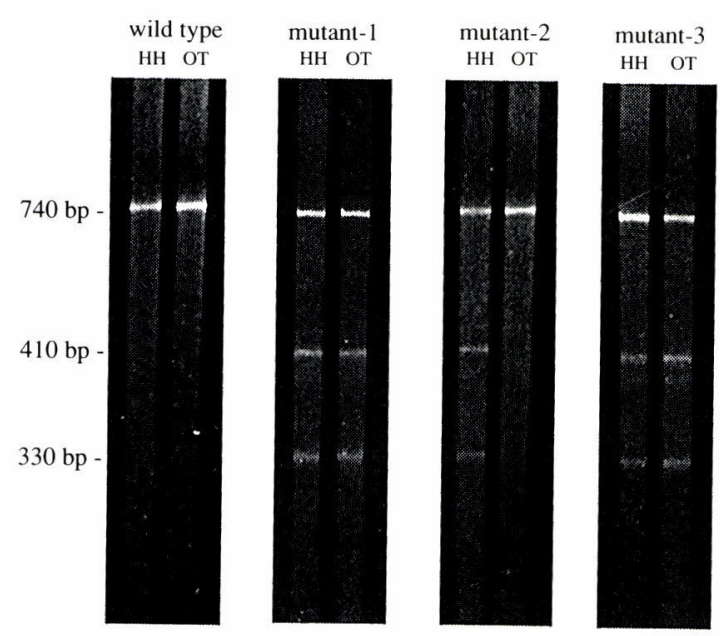

Fig. 2. Non-radioactive chemical mismatch in mutant-1, -2 , and -3 . While no cleavage occurred in wild-type homoduplex, cleaved fragments are observed in both $\mathrm{HH}$ (hydroxylamine hydrochloride treatment) and OT (osmium tetroxide treatment) lanes in mutant-1 (transversion of "T:A" to "G:C"). Cleaved fragments are seen only in the $\mathrm{HH}$ lane in mutant-2 (deletion of "C:G"). In mutant-3, cleaved fragments are observed in both $\mathrm{HH}$ and OT lanes ("G:C" and "T:A" deletion). 


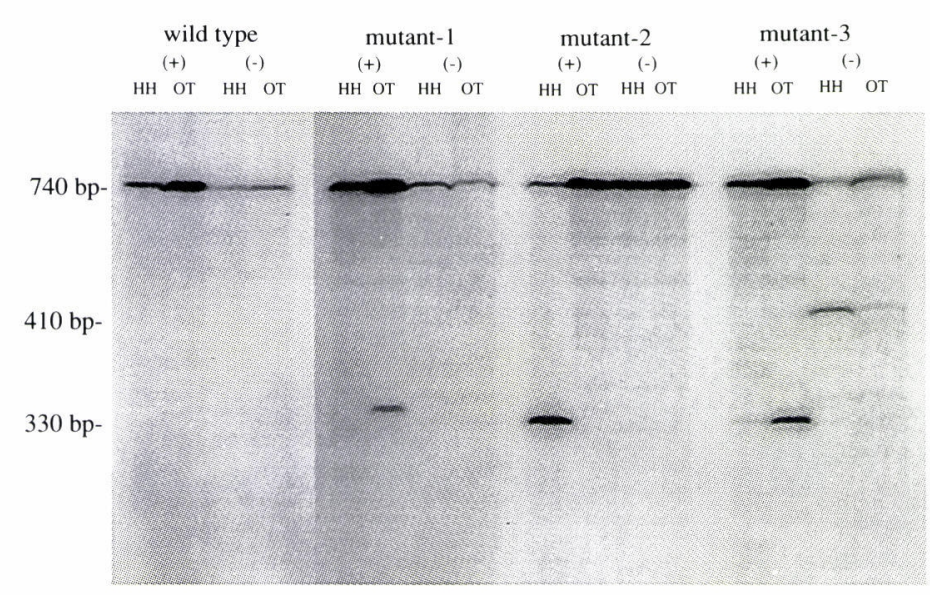

Fig. 3. Radioactive chemical mismatch analysis. There are four lanes for each sample. From left to right, plus-strand (+) with hydroxylamine hydrochloride treatment $(\mathrm{HH})$, plus-strand $(+)$ with osmium tetroxide treatment (OT), minus-strand (-) with $\mathrm{HH}$ treatment, and minus-strand $(-)$ with OT treatment. Wild type: homoduplex formed between differently prepared wild-type cDNAs. Mutants 1-3: Heteroduplexes formed between wild-type and mutant- $1,-2$ and -3 , respectively. No cleavage occurred in wild-type homoduplexes. In mutant-1, one cleaved fragment appeared in the (+)-strand OT lane. Also, one cleaved fragment showed up in the (+)-strand $\mathrm{HH}$ lane in mutant-2, and two cleaved fragments appeared in the (+)-strand OT and (-)-strand HH lanes of mutant-3.

shown). Several homoduplexes formed between independently amplified wildtype cDNA's were tested without yielding cleavage. One example of mismatch analysis with wild-type homoduplexes is shown in Fig. 2. In mutant-1, two cleaved fragments ( 410 and $330 \mathrm{bp}$ ) were seen with both HH and OT treatments, which indicates that the heteroduplexes between wild-type and mutant-1 have mismatching involving " $\mathrm{T}$ " and "C", and their location is approximately $330 \mathrm{bp}$ from the annealing site of primer-3 or 4 (see Fig. 4). Similarly, mutant-2 has cleaved fragments only in the $\mathrm{HH}$ lane, while mutant-3 has cleaved fragments in both lanes. These indicate that mutant-2 has a mismatched " $\mathrm{C}$ " without involvement of "T", while mutant-3 has a mismatched "T" and "C". Unexpectedly, the size of resulting fragments turned out to be very similar, which indicates that 
1) Non-radioactive method

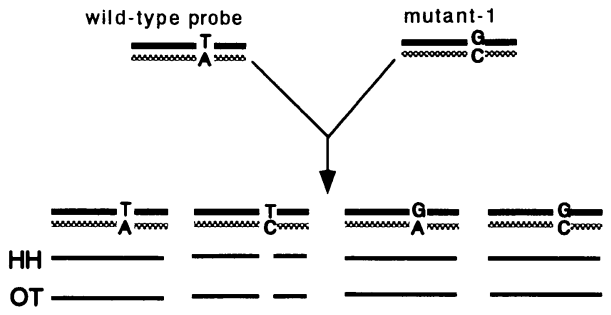

2) Radioactive method
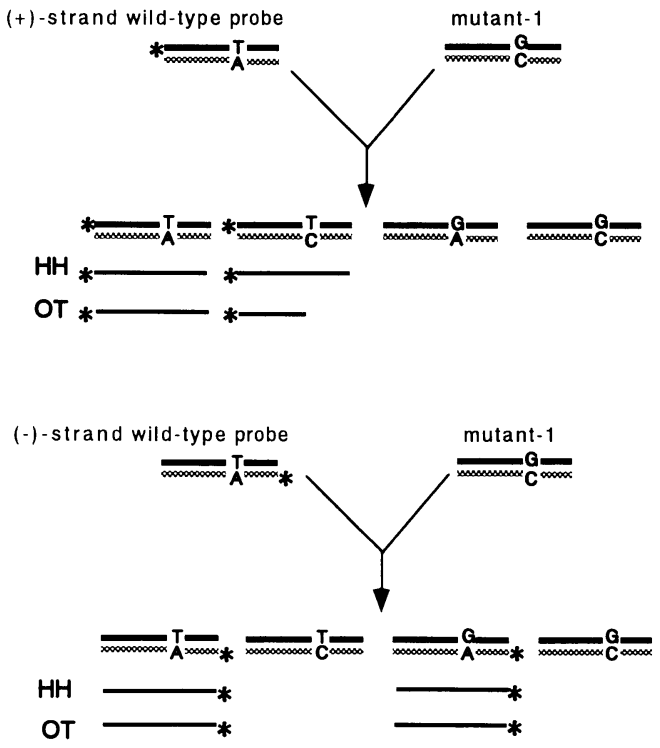

Fig. 4. Schematical illustration of mismatch analysis between wildtype and mutant-1. In the non-radioactive method, three differentsized fragments appear in both hydroxylamine hydrochloride $(\mathrm{HH})$ and osmium tetroxide (OT) lanes, since all cleaved fragments are visualized by ethidium bromide in this method. When strand-specifically radiolabeled wild-type probes are used, however, only one cleaved fragment appears in the (+)-strand OT lane on autoradiography.

these mutants have point mutations or small deletions in closely located regions of exon 3 of HPRT cDNA. 

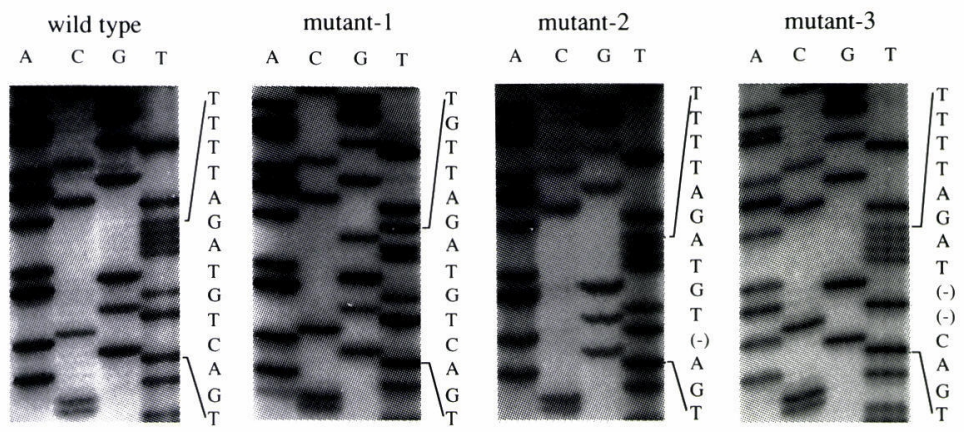

Fig. 5. Modified direct sequencing of the focused region. A "T" to "G" transversion is observed in mutant-1. A deletion of " $\mathrm{C}$ " is observed in mutant-2. A two-base deletion of "TG" is observed in mutant-3.

\section{Radioactive Mismatch Analysis (Fig. 3)}

Cleavage products were not observed in the control homoduplex where the radiolabeled wild-type was melted and re-annealed to a variety of "cold" wildtype PCR products. Cleavage products appeared in heteroduplexes formed between the wild-type and each of the mutant cDNAs. A single $330 \mathrm{bp}$ fragment was detected in plus-strand OT lane of mutant-1, which indicates the presence of a mismatched " $\mathrm{T}$ " in the plus-strand located approximately $330 \mathrm{bp}$ from the annealing site of primer-3. Similarly, treatment of the heteroduplex formed between plus-strand wild-type and minus-strand mutant-2 with $\mathrm{HH}$ produced a cleavage product, which indicates the presence of a mismatched " $\mathrm{C}$ " in the same region of the plus-strand. Finally, analysis of mutant-3 indicated the presence of both a mismatched " $\mathrm{T}$ " in the plus-strand and " $\mathrm{C}$ " in the minus-strand. These results indicate that mutant-3 has mutations involving two bases.

Schematic explanation of non-radioactive and radioactive mismatch analyses between wild-type and mutant-1 is shown in Fig. 4 .

\section{Sequencing (Figs. 5 and 6)}

The PCR products purified by the method mentioned above were sufficient to consistently yield "clean" sequencing information by the dideoxy method. Snap-cooling (11) was effective in preventing reassociation of the denatured PCR product. Treatment of the reaction with terminal deoxynucleotidyl transferase 


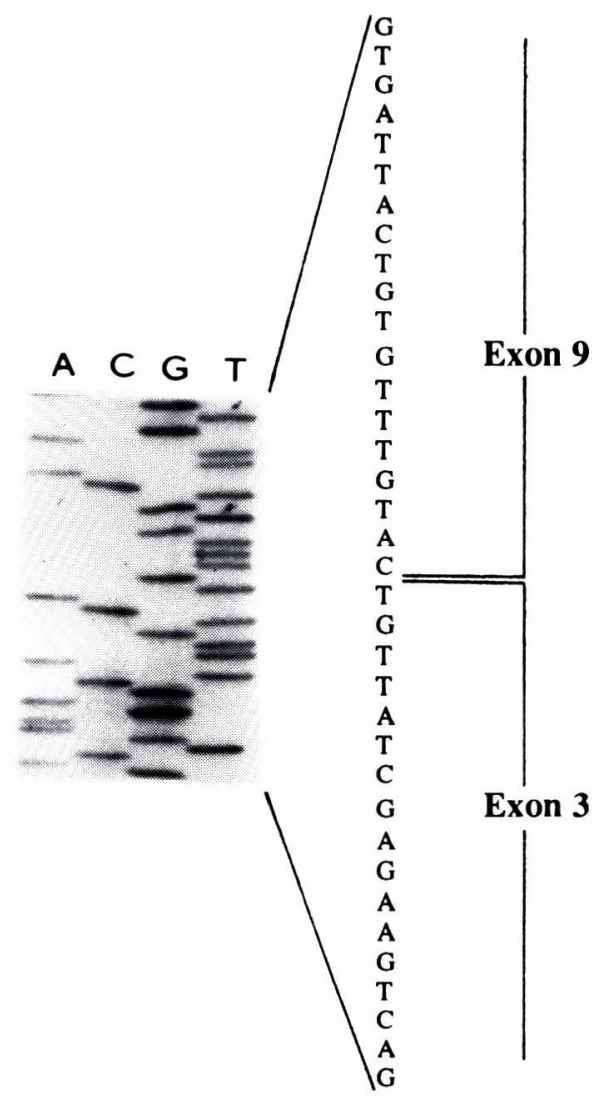

Fig. 6. Direct sequencing of the small fragment generated from mutant-3. In addition to the two base-pair deletion in exon 3, exons 4, $5,6,7$, and 8 are deleted.

(USB) worked dramatically to eliminate banding artifacts which frequently occur when PCR products are sequenced (10). One of three primers designed to span the cDNA for sequencing purposes was used to sequence the exon 3 region of mutants $-1,-2$, and -3 (Fig. 5). Mutant-1 has a T:A to G:C transversion at position of 381 . Mutant-2 has a deletion of G:C at position of 372, while mutant-3 has deletion of G:C and T:A at position of 373-374. These results are consistent with our interpretation of the data obtained by the mismatch analysis. In addition, sequencing data of the smaller fragment of mutant-3 indicated that in addition to a two-base pair deletion in exon 3 , this small fragment was devoid of sequences for exons 4,5 , 6, 7, and 8 (Fig. 6). 


\section{DISCUSSION}

The idea of amplifying the first strand of cDNA generated by reverse transcription of mRNA (12) made possible the mutational analysis of cDNA from extremely small quantities of starting materials, such as a crude lysate equivalent to a few cells (13) or the precipitated cells themselves (4). This is especially important in studies involving primary cells with a finite life span, such as HSF cells. In this one-step first strand cDNA synthesis from cell pellets of mutant-3, an altered size of cDNA appeared unexpectedly. The nature of this "new" fragment is not yet clear, but it has been reported that point mutations near intronexon boundaries can often cause aberrations in the splicing processes resulting in deletions by exon skipping (14). As a consequence, the splice site mutations can alter the size of the processed mRNA despite normal genomic Southern blot patterns (5).

The use of mismatch analysis to detect point mutations was first reported by Myers et al. $(15,16)$ using DNA:RNA hybrid molecules. Cotton et al. (8) improved this method considerably by using DNA:DNA hybrids and osmium tetroxide and hydroxylamine hydrochloride to modify specific bases prior to chemical cleavage. In addition to its high sensitivity, mismatch analysis has the capacity to precisely locate the position of mutations, thus enabling one to focus the sequencing to a smaller region. There are two other frequently used methods to detect point mutations in a given DNA fragment. One is single-strand conformation polymorphism (SSCP) (17) and the other is denaturing gradient gel electrophoresis (DGGE) (18). Although SSCP and DGGE can also readily detect point mutations; both of them lack positional information on the detected mutations. As a consequence, when one analyzes DNA sequences by SSCP or DGGE, one must sequence the entire length of the fragment to determine the precise molecular basis of each mutation. In addition, it has been claimed that SSCP and DGGE can be applied to fragments up to $400 \mathrm{bp}$ and $1,000 \mathrm{bp}$ in length, respectively $(17,18)$. In contrast, fragments larger than $1 \mathrm{~kb}$ can be analyzed by chemical mismatch analysis. According to Condie et al. (19), the sensitivity of each technique to detect point mutations is $90 \%$ for SSCP, $88 \%$ for constant denaturant gel electrophoresis (CDGE) and $100 \%$ for chemical mismatch analysis.

Although our PCR/mismatch protocol appears quite straight forward, this technique must cope with potential sequence changes that may result from the error-rate of the Taq DNA polymerase $(20,21)$. Krawczak et al. (22) have attempted to calculate the replication errors induced by extensive PCR amplifica- 
tion with the Taq DNA polymerase. Reiss et al. (23) tried to estimate the effect of replication errors in the mismatch analysis of PCR-amplified DNA. If their calculations were applied to our PCR scheme, approximately $57 \%$ of the final $740 \mathrm{bp}$ PCR product would not contain replication errors, which is adequate to maintain the sensitivity of our mismatch protocol, despite the tendency of the replication errors induced by the Taq enzyme to cluster at specific positions in a given DNA sequence (21). Actually, no false-positive signals were detected in our experiments, although several homoduplexes formed between independently amplified wild-type cDNA's were used for mismatch analysis.

The mismatch technique has relied primarily on a variety of radiolabeling techniques (8,24-26). Radiolabeling techniques (1) are time-consuming, (2) require special protocols and facilities, and (3) are constantly ongoing processes because of the short half-lives of radioactive probes. Our non-radioactive method is not only simple but also is as sensitive as the radioactive method if more than 500 ng of DNA is used. Furthermore, the non-radioactive method can potentially detect all types of mutation (deletion, base change, and insertion), while the radioactive technique cannot detect insertion mutations if only wild-type DNA is labeled. We believe that this approach is particularly useful as a screening tool to rapidly localize and identify small mutations when a large number of mutants are being analyzed.

\section{ACKNOWLEDGEMENTS}

We are indebted to Miss Chizuko Nonaka for her technical assistance and preparation of this manuscript. This work was supported by the U. S. Department of Energy and Los Alamos Laboratory Directed Research \& Development Funds.

\section{REFERENCES}

1. Vrieling, H., Simons, J.W.I.M. and Zeeland, A. A. (1988): Nucleotide sequence determination of point mutations at the mouse HPRT locus using in vitro amplification of HPRT mRNA sequences. Mutat. Res., 198, 107-113.

2. Davidson, B. L., Palella, T. D. and Kelley, W. N. (1988): Human hypoxanthine-guanine phosphoribosyltransferase: a single nucleotide 
substituion in cDNA clones isolated from a patient with Lesh-nyhan syndrome (HPRT Midland). Gene, 68, 85-91.

3. Simpson, D., Crosby, R. M. and Skopek, T. R. (1988): A method for specific cloning and sequencing of human HPRT cDNA mutation analysis. Biochem. Biophys. Res. Commun., 151, 487-492.

4. Yang, J. L., Maher, V. M. and McCormick, J. J. (1989): Amplification and direct nucleotide sequencing of cDNA from the lysate of low numbers of diploid human cells. Gene, 83, 347-354.

5. Recio, L., Cochrane, J., Simpson, D., Skopek, T. R., O'Neill, J. P., Nicklas, J. A. and Albertini, R. J. (1990): DNA sequence analysis of in vivo HPRT mutation in human T lymphocytes. Mutagenesis, 5, 505-510.

6. Noyes, E. (1979): Establishment of proliferating human epithelial cells in vitro from cell suspension of neonatal foreskin. TCA Manual, 5, 1173-1175.

7. Jolly, D. J., Okayama, H., Berg, P., Esty, A. C., Filpula, D., Bohlen, P., Johnson, G. G., Shively, E., Hunakapillar, T. and Friedman, T. (1983): Isolation and characterization of a full-length expressible cDNA for human hypoxanthine phosphoribosyltransferase. Proc. Natl. Acad. Sci. USA, 80, 477-481.

8. Cotton, R. G. H., Rodrigues, N. R. and Campbell, R. D. (1988): Reactivity of cytocine and thymine in single-base-pair mismatches with hydroxylamine and osmium tetroxide and its application to the study of mutations. Proc. Natl. Acad. Sci. USA, 85, 4397-4401.

9. Grompe, M., Muzny, D. M. and Caskey, C. T. (1989): Scanning detection of mutation in human ornithine transcarbamolase by chemical mismatch cleavage. Proc. Natl. Acad. Sci. USA, 86, 5888-5892.

10. Fawcett, T. W. and Bartlett, G. (1990): An effective method for eliminating "artifact banding" when sequencing double-stranded DNA templates. Biotechniques, 7, 46-48.

11. Casanova, J. L., Pannetier, C., Jaulin, C. and Kourilsky, P. (1990): Optimal conditions for direct sequencing double-stranded PCR products with Sequenase. Nucleic Acids Res., 18, 4028.

12. Veres, G., Gibbs, R. A., Schereer, S. E. and Caskey, C. T. (1987): The molecular basis of the sparse for mouse mutation. Science, 237, 415-417.

13. Sambrook, J., Fritsch, E. F. and Maniatis, T. (1989): Molecular cloning. A laboratory Manual (2nd Ed.), Cold Spring Harbor Laboratory Press, p14.33.

14. Weil, D., Bernard, M., Combates, N., Wirtz, M. K., Hollister, D. W., Steinmann, B. and Ramirez, F. (1988): Identification of mutations that cause exon skipping during collagen pre-mRNA splicing in an EhlersDanlos syndrome variant. J. Biol. Chem., 263, 8561-8564.

15. Myers, R. M., Lumelsky, N., Lerman, L. S. and Maniatis, T. (1985): Detection of single base substitutions in total genomic DNA. Nature, 313, 495498. 
16. Myers, R. M., Larin, Z. and Maniatis, T. (1985): Detection of single base substitutions by ribonuclease cleavage at mismatches in RNA:DNA duplexes. Science, 230, 1242-1246.

17. Orita, M., Iwahana, H., Kanazawa, H., Hayashi, K. and Sekiya, T. (1989): Detection of polymorphisms of human DNA by gel electrophoresis as singlestrand conformation polymorphisms. Proc. Natl. Acad. Sci. USA, 86, 27662770.

18. Fischer, S. G. and Lerman, L. S. (1983): DNA fragments differing by single base-pair substitutions separated in denaturing gradient gels: Correspondence with melting theory. Proc. Natl. Acad. Sci. USA, 80, 1579-1583.

19. Condie, A., Eeles, R., Borresen, A. L., Coles, C., Cooper, C. and Prosser, J. (1993): Detection of point mutations in the p53 gene: Comparison of singlestrand conformation polymorphism, constant denaturant gel electrophoresis, and hydroxylamine and osmium tetroxide techniques. Hum. Mutat., 2, 58-66.

20. Tindall, K. R. and Kunkel, T. A. (1988): Fidelity of DNA synthesis by the Thermus aquaticus DNA Polymerase. Biochemistry, 27, 6008-6013.

21. Keohavong, P. and Thilly, W. G. (1989): Fidelity of DNA polymerase in DNA amplification. Proc. Natl. Acad. Sci. USA, 86, 9253-9257.

22. Krawczak, M., Reiss, J., Schmidtke, J. and Rosler, U. (1989): Polymerase chain reaction: replication errors and reliability of gene diagnosis. Nucleic Acids Res., 17, 2197-2201.

23. Reiss, J., Krawczak, M., Schloesser, M., Wagner, M. and Cooper, D. N. (1990): The effect of replication errors on the mismatch analysis of PCRamplified DNA. Nucleic Acids Res., 18, 973-978.

24. Gibbs, R. A. and Caskey, C. T. (1987): Identification and localization of mutations at the Lesh-Nyhan locus by ribonuclease A cleavage. Science, 236, 303-305.

25. Mondanton, A. J., Green, P. M., Giannelli, F. and Bentley, D. R. (1989): Direct detection of point mutations by mismatch analysis: Application to haemophilia B. Nucleic Acids Res., 17, 3347-3358.

26. Saleeba, J. A. and Cotton, R. G. H. (1991): 35S-labelled probes improve detection of mismatched base pairs by chemical cleavage. Nucleic Acids Res., $19,1712$. 